\title{
Diffusion of fluorine on and between graphene layers
}

\author{
A. Sadeghi, ${ }^{1}$ M. Neek-Amal, ${ }^{2,3,{ }^{*}}$ G. R. Berdiyorov,${ }^{4}$ and F. M. Peeters ${ }^{3}$ \\ ${ }^{1}$ Department of Physics, Shahid Beheshti University, G. C., Evin, 19839, Tehran, Iran \\ ${ }^{2}$ Department of Physics, Shahid Rajaee Teacher Training University, Lavizan, Tehran 16785-136, Iran \\ ${ }^{3}$ Department of Physics, University of Antwerpen, Groenenborgerlaan 171, B-2020 Antwerpen, Belgium \\ ${ }^{4}$ Qatar Environment and Energy Research Institute, Qatar Foundation, P.O. Box 5825, Doha, Qatar \\ (Received 6 May 2014; revised manuscript received 3 December 2014; published 8 January 2015)
}

\begin{abstract}
Using first-principles calculations and reactive force field molecular dynamics simulations, we study the structural properties and dynamics of a fluorine $(\mathrm{F})$ atom, either adsorbed on the surface of single layer graphene (F/GE) or between the layers of AB stacked bilayer graphene (F@ bilayer graphene). It is found that the diffusion of the $\mathrm{F}$ atom is very different in those cases, and that the mobility of the $\mathrm{F}$ atom increases by about an order of magnitude when inserted between two graphene layers. The obtained diffusion constant for F/GE is twice larger than that experimentally found for gold adatom and theoretically found for $C_{60}$ molecule on graphene. Our study provides important physical insights into the dynamics of fluorine atoms between and on graphene layers and explains the mechanism behind the separation of graphite layers due to intercalation of $\mathrm{F}$ atoms.
\end{abstract}

DOI: 10.1103/PhysRevB.91.014304

PACS number(s): 66.10.C- $66.30 .-\mathrm{h}$

\section{INTRODUCTION}

Fluorinated graphene (fluorographene) has been attracting considerable interest in recent years [1-4] because of its unique physical, chemical, and mechanical properties [5-10]. Once adsorbed on the surface of graphene (GE), fluorine (F) atoms form a covalent bond with carbon (C) atoms changing their hybridization [1] from $s p^{2}$ to $s p^{3}$. This functionalization in turn tunes the electronic transport properties of GE by opening a nonzero band gap, overcoming the main drawback of practical applications of pristine GE in electronic devices [5,11]. Theory predicts the band gap of fluorinated GE to be in the range of $0-3 \mathrm{eV}$ depending on the degree of fluorination, and the full fluorographene behaves as an insulator with a band gap of approximately $3.1 \mathrm{eV}$ [7-9]. This property of fluorographene can also be used by patterning nanorods and quantum dots into pristine GE $[12,13]$ as well as channels for confining electrons [14]. Theory also predicts the possibility of fluorination of bilayer GE, where the weak van der Waals ( $\mathrm{vdW}$ ) interaction between the GE layers are converted into much stronger covalent bonds that stabilize the structure [15]. The structural properties of the resulting bilayer fluorographene are between those of monolayer fluorographene and diamond; other characteristics are closer to those of initial fluorographene $[16,17]$. Fluorination has advantages over conventional oxidation and hydrogenation when considering the engineering of graphene's band structure using chemical functionalization [18]. For example, in contrast to hydrogenation, the process of fluorination is easy to control and the resulting fluorographene shows high chemical and temperature stability as compared to hydrogenated GE [1]. In contrast to GE oxides, which are highly inhomogeneous, fluorographene is formed by an ordered arrangement of fluorine atoms on both sides of the GE layer.

One of the well studied methods for the synthesis of GE is mechanical (ultrasonic) exfoliation of chemically modified graphite (see Ref. [19] for review). To date, oxidation has been successfully implemented to modify graphite with high

*neekamal@srttu.edu yield and large throughput [20-23]. However, the oxidation process results in the formation of different oxygen-containing functional groups, which are difficult to replace or to fully remove; invasive chemical and thermal treatment of them generates structural defects on the surface of GE [24-26], which degrade the unique electronic properties of GE. In this context, fluorination is also well known to modify graphite with high yield and large throughput [27-29]. In contrast to the deoxidation process, fluorine atoms can be effectively removed retaining graphenes' carbon skeleton [7]. For effective GE fabrication based on sonochemical exfoliation of graphite, a uniform distribution of intercalated fluorine atoms is required in order to weaken the $\mathrm{vdW}$ interaction between the adjacent GE sheets [30].

Despite the increasing effort in studying graphene-fluorine complexes, no theoretical reports are available in the literature about the dynamics of $\mathrm{F}$ atoms between GE layers, whereas their dynamics under microwave and sonication is crucial for the graphite exfoliation process. Therefore, our aim in this work is to better understand the physical features of fluorine diffusion inside bilayer GE. First, we use density functional theory (DFT) to calculate the energy barrier (as well as the energy landscape) for the motion of a fluorine atom on top of a monolayer GE or within bilayer GE. Then we perform molecular dynamics (MD) simulations to study the dynamics of the $\mathrm{F}$ atom between bilayer graphene. In agreement with the prediction based on the DFT results, our reactive MD simulations show qualitative changes in the dynamics of the $\mathrm{F}$ atom when placed between graphene layers: (i) its migration starts at a temperature considerably lower than the one necessary for its motion on monolayer GE, and (ii) the atom travels over longer distances at the given temperature inside bilayer GE due to "hopping from one layer to the other."

The paper is organized as follows. Section II presents the DFT calculations for the structural properties of $\mathrm{F}$ atoms either adsorbed on the surface of single layer graphene or intercalated into bilayer graphene. In Sec. III, we discuss the dynamics of the $\mathrm{F}$ atom in both situations using the reactive MD simulations. Section IV summarizes our findings. 
TABLE I. Bond length and angles, Bader charge on the F atom, and binding energy per F atom from GGA-DFT for the C-F bond in the chair structure of fluorographene, single F/GE and single F@ bilayer GE, all at their most stable configuration.

\begin{tabular}{|c|c|c|c|c|c|c|}
\hline & \multicolumn{2}{|c|}{ Bond length $(\AA)$} & \multicolumn{2}{|c|}{ Angle $\left(^{\circ}\right)$} & \multirow[b]{2}{*}{ Charge of $F(e)$} & \multirow[b]{2}{*}{ Binding energy (eV/atom) } \\
\hline & DFT & Ref. [33] & F-C-C & C-C-C & & \\
\hline Fluorographene & 1.38 & 1.38 & 109.3 & 109.6 & -0.72 & -3.31 \\
\hline F/graphene & 1.56 & $1.56^{\mathrm{a}}$ & 101.9 & 115.8 & -0.57 & -2.51 \\
\hline F@bilayer GE & 1.52 & - & 98.4 & 117.8 & -0.60 & -1.18 \\
\hline
\end{tabular}

${ }^{\mathrm{a}}$ For the lowest reported concentration, i.e., $\mathrm{C}_{16} \mathrm{~F}$.

\section{DFT CALCULATIONS}

\section{A. Computational details}

In our DFT first-principles calculations, the cores are described with the projector-augmented wave (PAW) method [31] as implemented in the VASP code [32]. The valence electron wave function is expanded using a plane-wave basis set with an energy cutoff of $700 \mathrm{eV}$. For the exchangecorrelation functional, the generalized gradient approximation (GGA) of Perdew, Burke, and Ernzerhof (PBE) [34] is used. A Gaussian smearing of width $0.1 \mathrm{eV}$ is used during the calculations but finally the energies are extrapolated to zero temperature. In all calculations, periodic images in the direction perpendicular to the layer surface are separated with a vacuum region of height larger than $20 \AA$. A dipole correction is applied in order to eliminate the dipole-dipole interactions in this direction. Using a $40 \times 40 \times 1 \mathrm{k}$ mesh, an optimum lattice constant $a=2.464 \AA$ and an interlayer distance of $3.24 \AA$ for $\mathrm{AB}$ stacking are obtained if vdW dispersion correction is included [35]. (Without the vdW correction, GGA overestimates this interlayer distance by almost $1 \AA$.) Having these geometrical parameters determined, we then make a rectangular supercell of size $3 \sqrt{3} a \times 5 a \simeq 12.80 \times 12.32 \AA^{2}$, which contains 60 carbon atoms per layer in order to minimize the interactions between the periodic images of the $\mathrm{F}$ atom. With this supercell, we use a $4 \times 4$ mesh to sample the momentum space, and an energy cutoff of $400 \mathrm{eV}$. In the case of bilayer GE, we check that the interlayer distance remains $3.24 \AA$ when far from the $\mathrm{F}$ atom. Spin-polarized calculations are performed because a single electron of the $\mathrm{F}$ atom remains unpaired. A force criterion of $0.02 \mathrm{eV} / \AA$ is used for geometry relaxation.

\section{B. Properties of the C-F bond}

First, it is worthwhile to compare the nature of a single C-F bond with the C-F bond in fully covered graphene by fluorine atoms (i.e., fluorographene). We see appreciable differences between these two cases. For instance, for the chair structure of fluorographene, i.e., when every carbon is bonded to one $\mathrm{F}$ atom, the $\mathrm{C}-\mathrm{F}$ bond length is $1.38 \AA$ in complete agreement with previous calculations; see, e.g., Ref. [36] where it was also shown that the bond length is the same for other possible structures with the same stoichiometry. The $\mathrm{C}-\mathrm{F}$ bond length is, however, longer by about $15 \%$ in the more stable configuration, i.e., when only a single $\mathrm{F}$ atom exists in the supercell, see Table I. Similar results for the F-C bond length (in the range of 1.55-1.57 $\AA$ ) have been found in recent DFT calculations $[37,38]$. A very close value $(1.56 \AA)$ has been reported for low concentration of $F$ in graphite $\left(\mathrm{C}_{16} \mathrm{~F}\right)$ [33].
This can be explained in terms of orbital hybridizations. In a highly fluorinated GE which is significantly buckled, the carbon atoms have $s p^{3}$ hybridization and the C-F bond is naturally covalent. In low fluorinated graphite, however, the hybridization of the carbon atom bonded to $\mathrm{F}$ is not perfectly $s p^{3}$. Note that the F-C-C angle in the latter case is $101.5^{\circ}$ while it is $109.5^{\circ}$ for $s p^{3}$ hybridization. Since the layer is buckled close to the $\mathrm{F}$ atom, the $\mathrm{C}-\mathrm{C}-\mathrm{C}$ angles close to that region deviate from the pure $120^{\circ}$ of $s p^{2}$ hybridization of pristine GE. For the same reason, the $\mathrm{C}-\mathrm{C}$ bond length between the $\mathrm{C}$ atom bonded to $\mathrm{F}$ and to the three adjacent carbons is $1.48 \AA$, which is between 1.42 and $1.55 \AA$ of graphite $\left(s p^{2}\right)$ and diamond $\left(s p^{3}\right)$, respectively.

We consider three important sites over a graphene layer: atop a carbon atom, which we call it top site (T), atop the center of a hexagon, which we call it hollow site $(\mathrm{H})$, and atop the center of a C-C bond, which we call it bridge-site (B). For absorption of an adatom with two valance electrons, e.g., an oxygen atom, the $\mathrm{B}$ site is preferred because the two electrons are shared with two carbons on the B site. In contrast, the F atom has one electron and it is therefore more likely that it bonds to the $\mathrm{T}$ site where it can share the single electron with the carbon atom underneath.

In order to examine this difference further, we show the distribution of the valence electron density around the C-F bonds in Fig. 1 for fluorographene (a) and F/GE (b). In the right panels, atomic structures are removed to help see the details of the electron density contours. By following the contour lines in the C-F bond region, it is seen that the electron density drops more rapidly in (b) compared to (a). This means that the covalent nature of the fluorographene is stronger than F/GE. In other words, more electrons are shared with $\mathrm{F}$ atoms when every $\mathrm{C}$ is bonded to a fluorine atom (i.e., for fluorographene). A quantitative measure of the charge transfer can be obtained by the Bader charge decomposition $[39,40]$ where two atoms are separated by an imaginary surface across which the gradient of the electron density vanishes. The smaller the charge transfer to $\mathrm{F}$, the weaker the electrostatic interaction between $\mathrm{F}$ and $\mathrm{C}$ and the longer the bond length (see Table I). The binding energy is calculated as the energy required to detach the $\mathrm{F}$ atom(s) from the system per $\mathrm{F}$ atom. The binding energy of F/GE is lower than for the fluorographene case. This will make the $\mathrm{F}$ atoms on or between GE layers more mobile as will be shown in the next sections.

It is worthwhile to mention that in both single layer and bilayer graphene adding a fluorine atom does not induce a net magnetic moment to the system. This is in contrast to adding a hydrogen atom. As discussed in Refs. $[13,37]$ for the adsorption of on a fluorine atom over a large graphene unit 

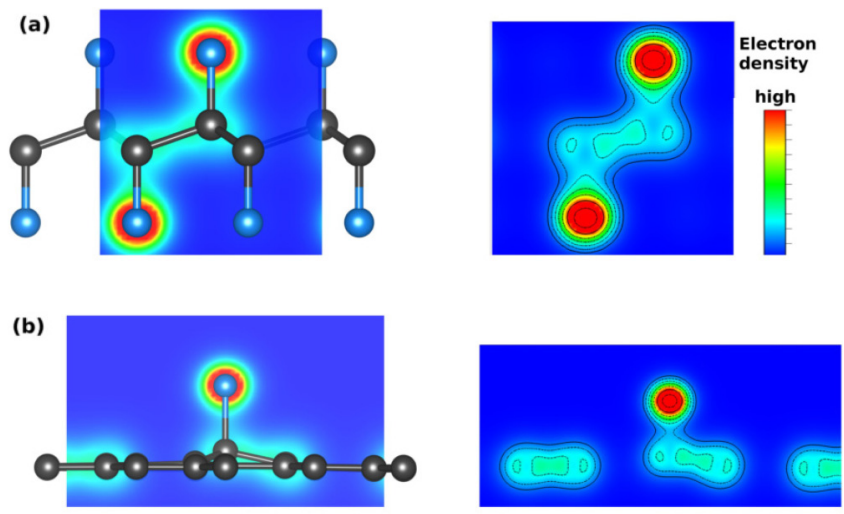

(c)
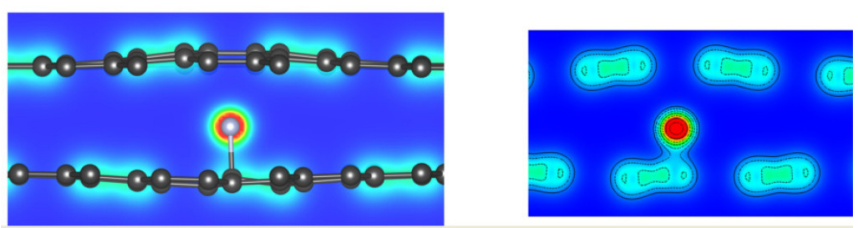

FIG. 1. (Color online) Valence electron density for (a) fluorographene, (b) single F on GE, and (c) single F between GE layers. Atomic structures are not shown on the right panels for better visualizing the details of electron density.

cell (larger than $3 \times 3$ ) the system is a nonmagnetic semimetal, which is attributed to the appearance of a vacancy like state. For example, it was found that in computations with a $3 \times 3$ unit cell (named as $\mathrm{CF}_{0.056}$ in Ref. [13]), the Fermi level shifts down into the valence bands of graphene and is about $0.75 \mathrm{eV}$ below the Dirac point, resulting in a hole dopant (for more details see Ref. [13]).

\section{Energy landscape}

The profile of the potential energy surface (PES) at zero temperature is helpful to understand the diffusion of an atom at finite temperature. Therefore we show in Fig. 2 the PES for the $\mathrm{F}$ atom on a single layer (a) and between two layers of GE (b) as described by DFT. Our sampling grid covers only a nonreducible fraction of one unit cell and then the whole PES
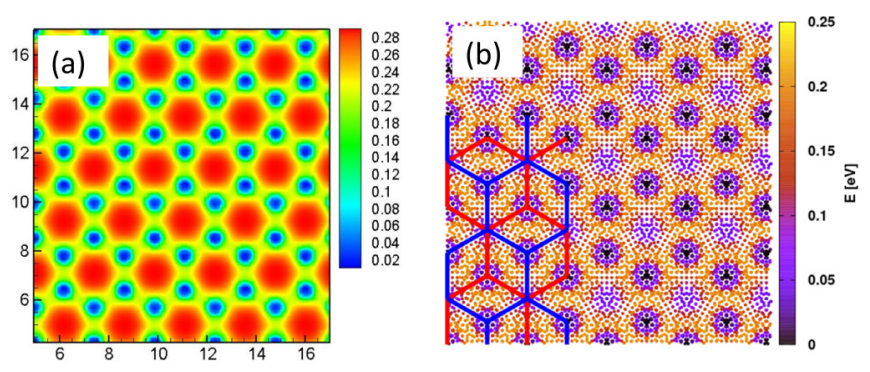

FIG. 2. (Color online) (a) Potential energy surface of F/GE in $\mathrm{eV}$. $\mathrm{F}$ is relaxed only in the $z$-direction while all $\mathrm{C}$ atoms are fully relaxed. The minimum energy (shifted to zero) occurs on top of the carbon atoms. (b) Same as (a) but for F@ bilayer GE in AB stacking. A model of bilayer GE shown by red and blue hexagons is overlaid to help the eye. The minimum energy which is shifted to zero occurs at the T-H sites. The vertical and horizontal axes have Angstrom units.
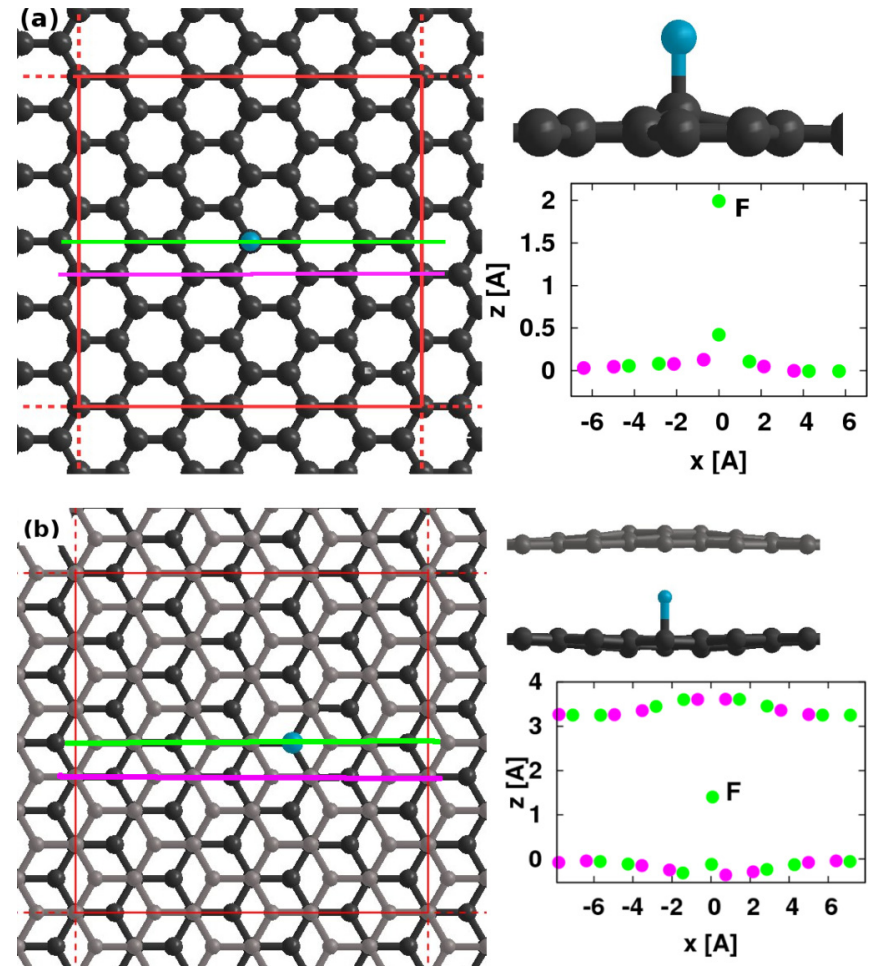

FIG. 3. (Color online) Top and side views of the minimum energy configuration of (a) F/GE and (b) inside bilayer GE, i.e., $\mathrm{T}$ and $\mathrm{T}-\mathrm{H}$ sites, respectively. In the bilayer case, one layer is shown in gray for clarity. The red boxes indicate the supercell borders. The $z$ position of the atoms along the green and purple lines are shown in the right column plots; the buckling of the layers are $+0.43 \AA$ in (a) and +0.36 and $-0.33 \AA$ in (b).

is obtained by symmetry operations. Note that the potential energy as a function of the lateral position $(x, y)$ of the $\mathrm{F}$ atom is evaluated after the atom finds its equilibrium position in the vertical direction. The carbon atoms are also relaxed resulting in a buckling of the GE layer(s) in the proximity of $F$ as shown in Fig. 3. In the latter figure, we have visualized the most stable geometries corresponding to the energy minima in Figs. 2(a) and 2(b), namely when the $\mathrm{F}$ atom is on $\mathrm{T}$ site (on top of a $\mathrm{C}$ atom) of a single layer GE or on T-H site [on $\mathrm{T}$ site of one layer and hollow site ( $\mathrm{H}$ site) of the other] of a bilayer GE, respectively. More geometrical properties are listed in Table I.

On the other hand, it is interesting to see how the potential energy varies when the $\mathrm{F}$ atom changes its position inside bilayer GE. This quantity versus the separation of $F$ from the frozen GE plane(s) is shown in Fig. 4. For the single layer, shown in Fig. 4(a), we put the F atom on top of a carbon atom (T), on a hollow $(\mathrm{H})$ or a bridge $(\mathrm{B})$ site. F atom on the $\mathrm{T}$ site has the lowest energy compared to the other two, hence during the diffusion it is expected that the $\mathrm{F}$ atom spends most of its time close to the $\mathrm{C}$ atoms at a constant height above the GE layer.

For the bilayer case, the $\mathrm{F}$ atom is moved from a $\mathrm{T}$ site on one layer to the $\mathrm{T}$ or $\mathrm{H}$ site on the other without changing its lateral position which is shown by $\mathrm{T}$ to $\mathrm{T}$ and $\mathrm{T}$ to $\mathrm{H}$ legends in Fig. 4(b), respectively. The $\mathrm{T}$ to $\mathrm{T}$ path is symmetric with respect to the middle of bilayer GE and the minimal energy is obtained when $\mathrm{F}$ is in the middle, i.e., at separation 

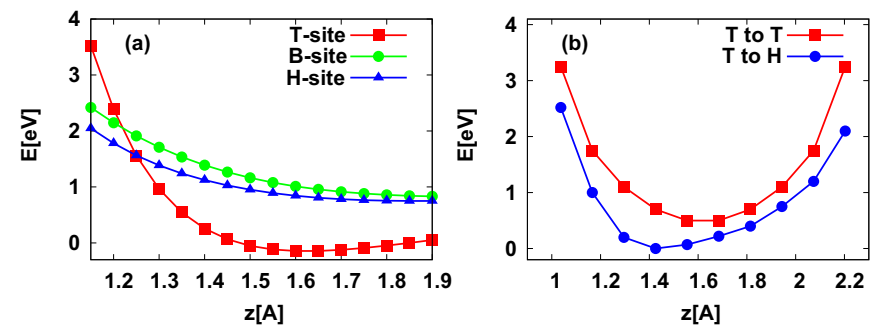

FIG. 4. (Color online) (a) Energy of a fluorine atom as a function of its distance to a frozen GE single layer when $\mathrm{F}$ is on top of a $\mathrm{C}$ carbon ( $\mathrm{T}$ site), hollow (H site) and bridge (B site). (b) Energy of a fluorine atom between two frozen GE layers as a function of its distance to the bottom layer, when the $\mathrm{F}$ atom moves from a $\mathrm{T}$ site on the bottom layer to the $\mathrm{T}$ or $\mathrm{H}$ sites on the top layer. The interlayer separation between unbuckled GE layers is $d=3.24 \AA$.

$d / 2=1.72 \AA$ from each C. In the more favorable configuration, which is along the $\mathrm{T}$ to $\mathrm{H}$ path, the equilibrium position is shifted towards the T site. Therefore, the $z$ position of the $\mathrm{F}$ atom diffusing between the layers would successively change by about half an $\AA$ by jumping and sticking to the opposite layers. Notice that by adding a single $\mathrm{F}$ over and between graphene layers, the system remains gapless [13].

\section{MOLECULAR DYNAMICS SIMULATIONS}

\section{A. Computational method}

In order to study the dynamical properties of $F$ over graphene and between graphene layers, we conduct MD simulations using reactive force-field ReaxFF, which is a general bond-order dependent potential that provides an accurate description of bond breaking and bond formation during chemical reactions [41]. The corresponding ReaxFF parameters for fluorographene can be found in our previous papers $[42,43]$.

As a representative example, we consider $\mathrm{AB}$ stacked bilayer GE (with 960 carbon atoms in each layer) with a fluorine atom between them. We implement periodic boundary conditions along the GE basal planes to avoid edge effects. The studied systems are first energy-optimized using the conjugate gradient method. When the system reaches equilibrium, the temperature is increased up to $2500 \mathrm{~K}$ at a rate of $20 \mathrm{~K} / \mathrm{ps}$ using an isothermal isobaric (NPT) ensemble with a Nose-Hoover thermostat for temperature and pressure control. When the desired temperature is reached, constant temperature MD simulations are conducted using the NPT ensemble. The damping constants for temperature and pressure are $0.25 \mathrm{fs}$ and $1 \mathrm{ps}$, respectively, and the time step was $0.25 \mathrm{fs}$ in all simulations. The results present in this manuscript are averaged over an ensemble of 3 different initial distributions of velocities of the atoms.

\section{B. Dynamics of F/graphene}

We first report F/GE results [see Figs. 5(a) and 5(b)]: as we have shown in the previous section and as was also shown in previous density functional calculations [5,6], once adsorbed on the surface of GE, fluorine atoms form covalent

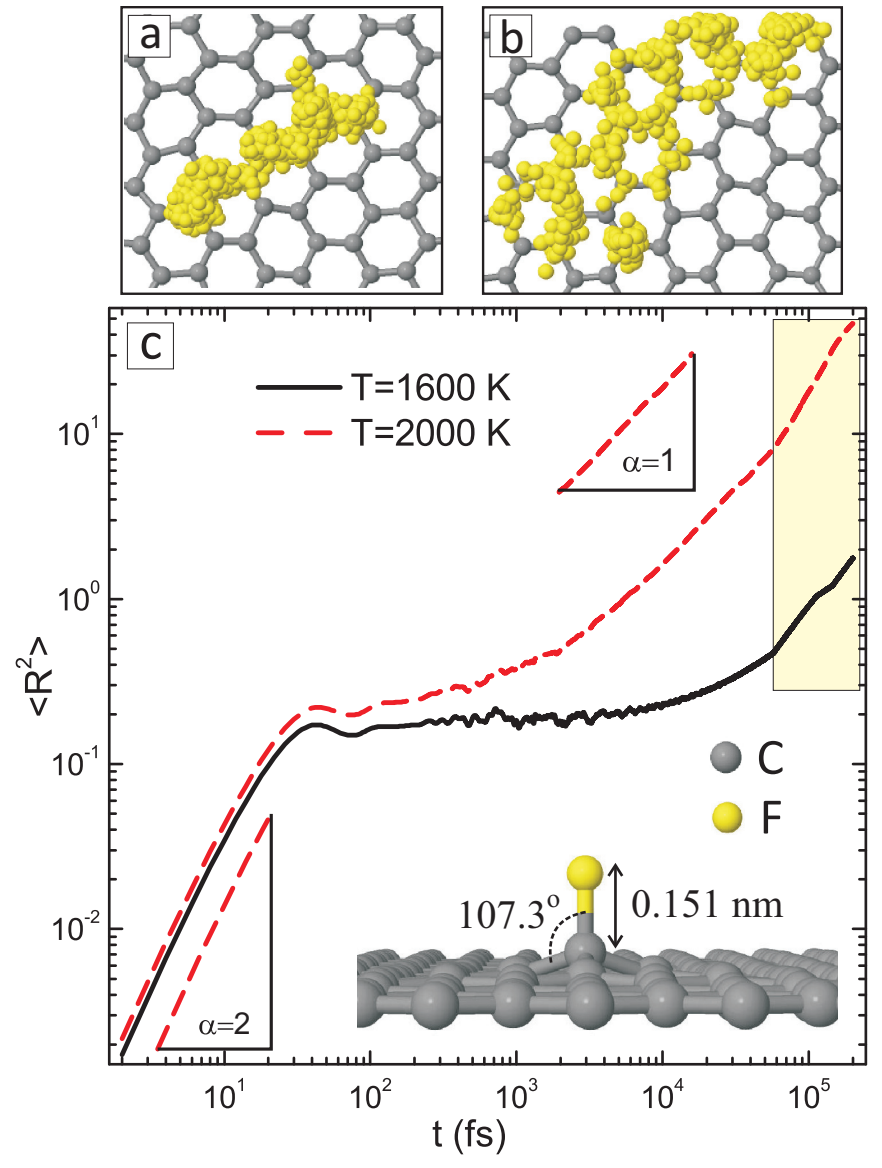

FIG. 5. (Color online) Superimposed consecutive snapshots (with time interval of $0.25 \mathrm{ps)}$ of a fluorine atom (yellow dots) migrating on the surface of monolayer GE (with a unit cell containing 960 carbon atoms) at (a) $T=2000$ and (b) $2400 \mathrm{~K}$. (c) Mean-square displacement $\left\langle R^{2}\right\rangle\left(\AA^{2}\right)$ as a function of time for the motion of the fluorine atom at $T=1600 \mathrm{~K}$ (solid black curve) and $T=2000 \mathrm{~K}$ (dashed red curve). The region that is used to estimate the diffusion coefficient is highlighted by the yellow area. Inset shows a side view of the equilibrium structure of the fluorine atom at zero temperature.

bonds with the carbon atoms. These carbon atoms change their hybridization from $s p^{2}$ to $s p^{3}$, resulting in the opening of a nonzero band gap. Our MD simulations also show covalent bonding of the $\mathrm{F}$ atom on GE with carbon-fluorine distance $1.51 \AA$, which is in reasonably good agreement with our DFT prediction (1.56 $\AA$, see Table I). The equilibrium structure of the system is shown in the inset of Fig. 5(c). It is seen from this figure that the $\mathrm{F}$ atom is adsorbed at the $\mathrm{T}$ site on top of a carbon atom, resulting in a considerable local change in the underlying planar GE. Such T site attachment of $\mathrm{F}$ atom was found in our DFT simulations to be stronger than on the B site (above the middle of carbon-carbon bonds) and $\mathrm{H}$ site (above the center of hexagon) attachment (see Fig. 4).

DFT calculations, shown in Fig. 2(a), for the variation of the total energy along the symmetry points (i.e., T, $\mathrm{H}$ and $\mathrm{B}$ sites) predict the migration path of the $\mathrm{F}$ atom to be along the edges of hexagons from the $\mathrm{T}$ site to the neighboring $\mathrm{T}$ site through a barrier of $\sim 0.13 \mathrm{eV}$ at the $\mathrm{B}$ site, which is equivalent to about $1560 \mathrm{~K}$. The latter temperature determines the threshold 
for diffusive motion. To show the surface migration process of the F atom, we plot in Fig. 5(a) superimposed consecutive snapshots of the calculated system configurations at $T=2000$ $\mathrm{K}$. It is seen from the trajectory of the F atom that it moves along the edges of the hexagon along which the migration barrier is the lowest. However, we observe only short-range diffusion of the $\mathrm{F}$ atom: in our $0.5 \mathrm{~ns}$ long simulations the maximal net displacement of the $\mathrm{F}$ atom was less than 10 GE sites. Fluctuations in the position path are due to thermal fluctuations and due to fluctuations of the graphene lattice. The distance traveled by the $\mathrm{F}$ atom increases with increasing temperature as shown in Fig. 5(b), where we plot the snapshots of the system at $T=2400 \mathrm{~K}$. Still, the F atom follows the path with the lowest energy barrier for its migration. Note, however, that desorption and consequent adsorption processes are also observed at this temperature range (not shown here). Figure 5(c) shows the mean square displacement $\left\langle R^{2}\right\rangle$ of the $\mathrm{F}$ atom at temperatures $T=1600 \mathrm{~K}$ (solid black curve) and $T=2000 \mathrm{~K}$ (dashed red curve) as a function of time, where $R^{2}=x^{2}+y^{2}$. Diffusion coefficient for the $\mathrm{F}$ atom is obtained from the mean square displacement in the large time regions [see the yellow region in Fig. 5(c)] as $D=0.002 \AA^{2} / \mathrm{ps}$ and $D=0.07 \AA^{2} / \mathrm{ps}$ for temperatures $T=1600$ and $2000 \mathrm{~K}$, respectively.

Thus a single $\mathrm{F}$ atom binds to GE covalently disturbing the $s p^{2}$ hybridization of the carbon atoms of GE at temperatures lower than $1560 \mathrm{~K}$. The migration of a single fluorine adatom on the surface of GE takes place at higher temperatures. The adatom moves predominantly along the edges of the hexagon where the barrier for its diffusion is minimal (see Fig. 2).

\section{Dynamics of F@bilayer graphene}

In this section, we study finite temperature dynamics of a fluorine atom between two graphene sheets. First, we note that the barrier height in case of bilayer GE found to be $0.1 \mathrm{eV}$ from DFT is about $20 \%$ less than that of $\mathrm{F}$ over single layer graphene. We consider a single $\mathrm{F}$ atom within AB-stacked bilayer GE (with 960 carbon atoms in each layer), the equilibrium structure of which is shown in Figs. 6(a) and 6(b). As seen from this figure, the $\mathrm{F}$ atom forms a covalent bond with the bottom layer on the $\mathrm{T}$ site (consequently on the $\mathrm{H}$ site of the top layer), resulting in a local expansion of the interplanar space of the GE layers. The C-F distance is found to be $1.45 \AA$, which is smaller than the $\mathrm{C}-\mathrm{F}$ for monolayer GE which is $1.51 \AA$. The maximum buckling of the top GE layer is $0.54 \AA$, which is slightly larger than the estimates from our DFT study (0.36 ̊, see Fig. 3). In our 500 ps long simulations, the threshold temperature for the migration of the $\mathrm{F}$ atom is about $1000 \mathrm{~K}$. The $\mathrm{F}$ atom migrates in the interplanar spacing distorting the GE layers locally. To trace the evolution of the F atom, we plot in Figs. 6(c) and 6(d) consecutive snapshots of the system, which shows diffusion pathways of the $\mathrm{F}$ atom inside the GE bilayer. From these two plots we can identify two qualitative differences in the dynamics of the $\mathrm{F}$ atom inside the bilayer GE as compared to its motion on top of monolayer GE (see Fig. 5): (i) the F atom moves between the two graphene layers by hoping from one GE layer to the other [Fig. 6(c)], and (ii) it does not follow the hexagonal trajectory
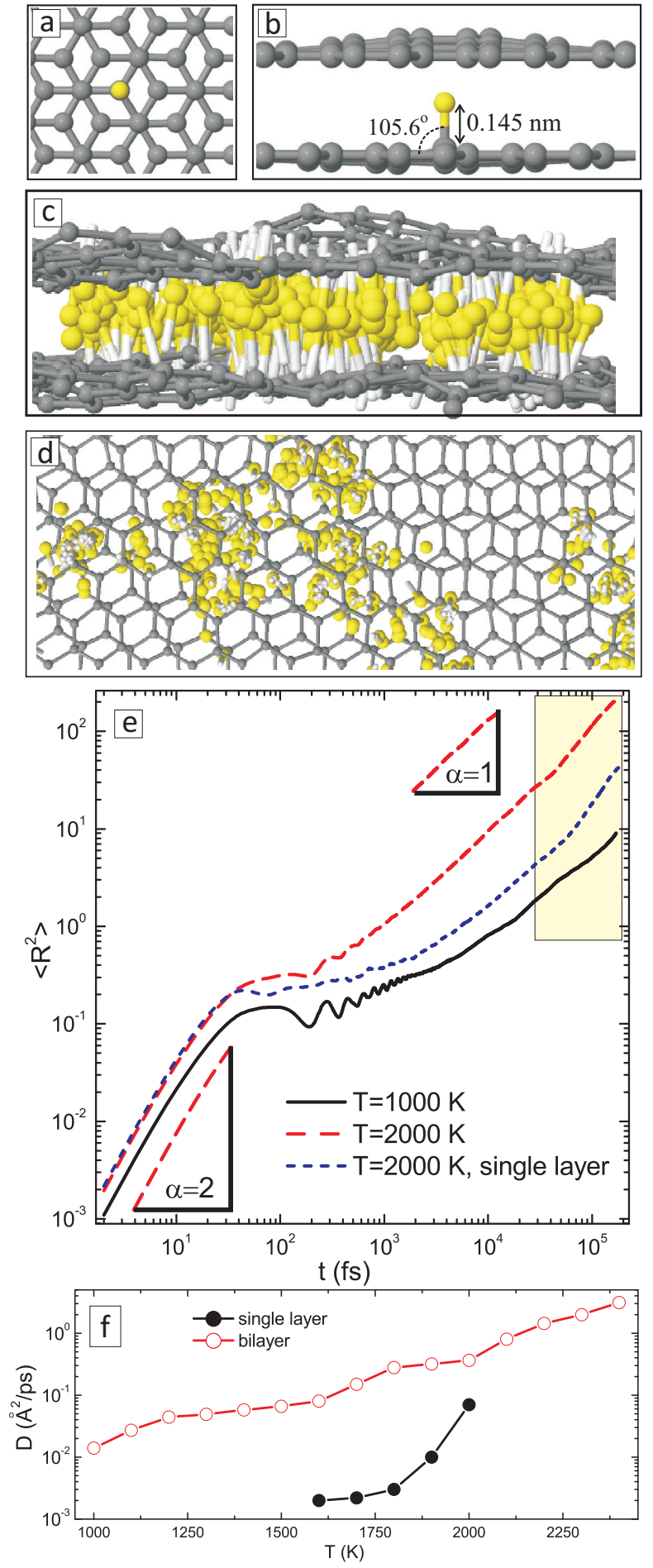

FIG. 6. (Color online) Top (a) and side (b) view of the equilibrium structure of a fluorine atom inside an $\mathrm{AB}$ stacked bilayer graphene (with 960 atoms in each layer). Side view (c) and top view (d) of consecutive snapshots of the system (over a time interval of $0.25 \mathrm{ps}$ ) at $T=2000 \mathrm{~K}$ illustrating dynamics of the $\mathrm{F}$ atom. Carbon atoms are shown only in the first frame. (e) Mean-square displacements $\left\langle R^{2}\right\rangle$ of the $\mathrm{F}$ atom at $T=1000 \mathrm{~K}$ (solid black curve) and at $T=2000 \mathrm{~K}$ (dashed red curve). Dotted blue curve shows the results when the $\mathrm{F}$ atom is attached on the surface of a monolayer GE. (f) Temperature dependence of the diffusion coefficient of the $F$ atom on top of the single layer graphene (solid circles) and inside the bilayer graphene (open circles). 

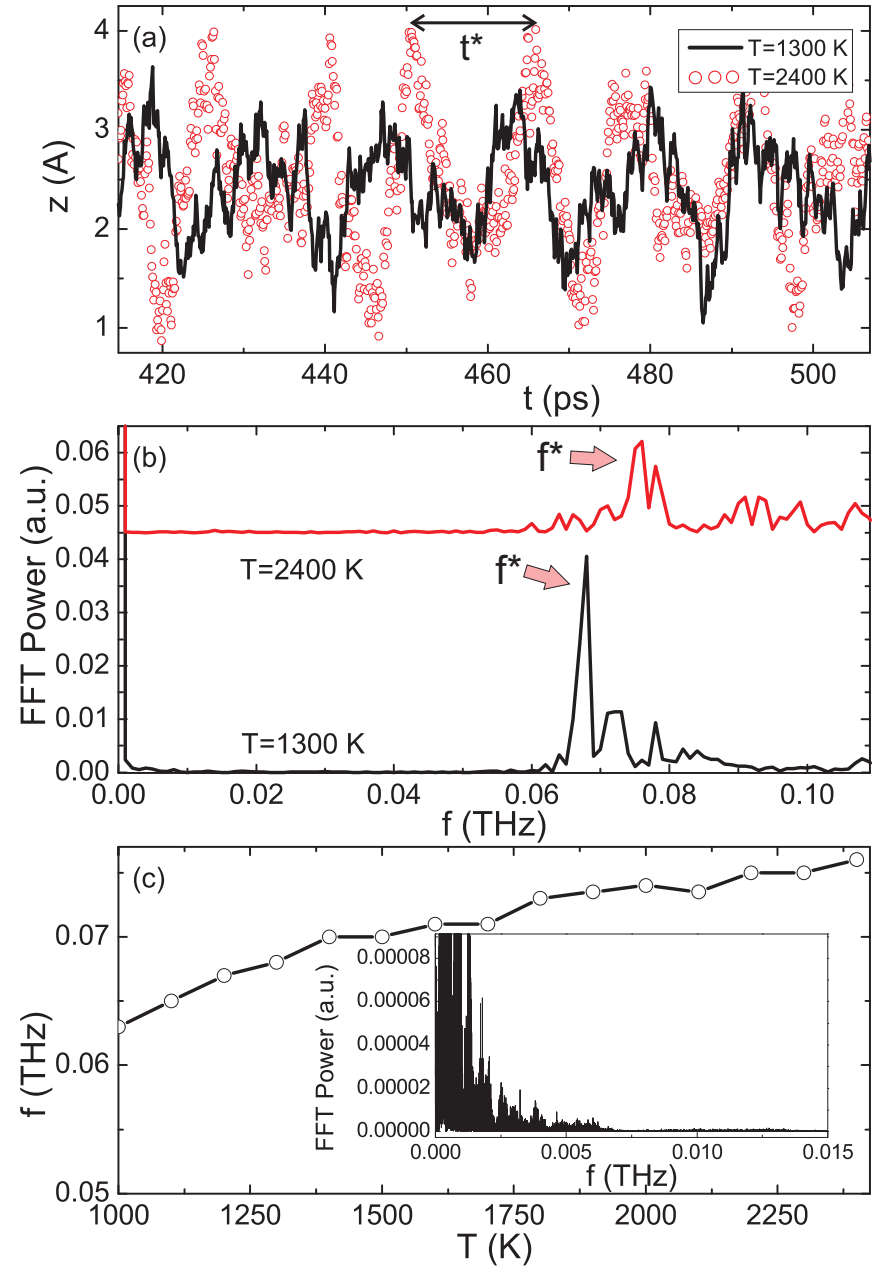

FIG. 7. (Color online) (a) $z$ coordinate of the F atom intercalating bilayer graphene at two different temperatures. (b) Fourier power spectrum of the time dependence of the $z$ coordinate of the $\mathrm{F}$ atom. The results for $T=2400 \mathrm{~K}$ are shifted by 0.045 . The arrows indicate the position of the characteristic frequency of the system $f^{*}$, which corresponds to the frequency of the "hopping" of the F atom between the graphene layers. (c) Temperature dependence of the characteristic frequency $f^{*}$. Inset shows the Fourier power spectrum of the $\mathrm{F}$ atom adsorbed on the surface of single layer graphene at $T=2000 \mathrm{~K}$.

[Fig. 6(d)] contrary to the monolayer GE system case. To illustrate this "hopping" process, we plot in Fig. 7(a) the time dependence of the $z$-coordinate of the $\mathrm{F}$ atom. We observe an oscillatory motion of the $\mathrm{F}$ atom between the graphene layers, characterized by a time period $t^{*}$. This periodicity is clearly shown in Fig. 7(b) where we plot the Fourier power spectrum of the $z(t)$ curves in Fig. 7(a). Clear peaks are observed in the spectra that correspond to the hopping process. As expected, this characteristic frequency $f^{*}$ increases with increasing temperature [see Fig. 7(c)]. Note, however, that no periodicity is observed in the motion of the $\mathrm{F}$ atom on the surface of single layer graphene, which is clearly seen from the Fourier spectrum of the $z(t)$ curve [see the inset of Fig. 7(c)].

To quantify the mobility of the $\mathrm{F}$ atom inside the bilayer GE, we show in Fig. 6(e) the mean square displacement $\left\langle R^{2}\right\rangle$ of the $\mathrm{F}$ atom as a function of time at $T=1000 \mathrm{~K}$ (solid black curve) and $T=2000 \mathrm{~K}$ (dashed red curve). Blue dotted curve in this figure shows the results when the $\mathrm{F}$ atom is attached to monolayer GE at $T=2000 \mathrm{~K}$. At small times $(t \leqslant 100 \mathrm{fs})$, the $\mathrm{F}$ atom shows the same $\left\langle R^{2}\right\rangle$ in both cases. However, the difference in $\left\langle R^{2}\right\rangle$ becomes significant at later times: the distance that the $\mathrm{F}$ atoms travels inside the bilayer GE becomes an order of magnitude larger than the one for the case of monolayer GE. Figure 6(f) shows the temperature dependence of the diffusion coefficient of the $\mathrm{F}$ atom when it is attached to the surface of single layer graphene (filled circles) and when intercalated inside the bilayer graphene (open circles). Notice that for $T<1800 \mathrm{~K}$, there is an order of magnitude difference, however, the mobility of the $\mathrm{F}$ atom attached to single layer graphene increases more steeply at higher temperatures before it is desorbed from graphene ( $T>2000 \mathrm{~K})$.

Finally, it is worthwhile to compare qualitatively the diffusion constant between graphene layers and above them. The different diffusion processes is mainly originated from different energy landscapes proposed by the single layer or bilayer to the $\mathrm{F}$ atom. Any diffusive motion can be described by the Langevin equation, which is written as

$$
\vec{r}(t)+\xi \overrightarrow{\dot{r}}+\vec{F}=\vec{\lambda}(t)
$$

where $\vec{F}$ is the force exerted on the $\mathrm{F}$ atom from the grephene layer(s), $\xi$ is the friction coefficient and corresponds to the mutual interaction between $\mathrm{F}$ and graphene layer(s), and $\lambda$ is a random force representing the finite temperature. Clearly, different force results in different trajectory and since diffusion constant is

$$
D=\frac{1}{3} \int\langle\vec{v}(t+\tau) \cdot \vec{v}(t)\rangle d \tau=\frac{1}{6 t}\left\langle|\vec{r}(t+\tau)-\vec{r}(t)|^{2}\right\rangle,
$$

which can be approximated by $D \sim \frac{k_{B} T}{\xi}$. Therefore the diffusion constant is inversely proportional to the friction coefficient. In F@bilayer, the mutual interaction is weaker (smaller $\xi$ ) thus we expect a larger diffusion constant.

\section{CONCLUSION}

Using first-principles calculations, we investigated how the potential energy of a single $\mathrm{F}$ atom changes when it scans different lateral sites on a GE layer or inside bilayer GE. In both cases, it turned out that the most favored configurations are obtained when the F atom is covalently bonded to only one carbon atom. We found that when the concentration of the $\mathrm{F}$ atoms is very low, as in the present case, the C-F bond is weaker than in the fully fluorinated case and thus the $\mathrm{F}$ atom is able to hop between different equilibrium positions. We then studied the dynamics of the $\mathrm{F}$ atom either trapped on single layer GE or between bilayer GE using reactive molecular dynamics simulations. We found that the diffusion of a single $\mathrm{F}$ atom between bilayer GE is larger than the same atom absorbed on a single-layer graphene, i.e., the diffusion constant of F@bilayer GE is one order of magnitude larger than that of F/single layer GE. The diffusion process in both cases is very different: the $\mathrm{F}$ atom above graphene diffuses 
along hexagonal paths hopping from one $\mathrm{T}$ side to the next $\mathrm{T}$ side of the nearest carbon atom, while the $\mathrm{F}$ atom between two graphene layers hops vertically from one graphene layer to the other and at the same time moves laterally from a $\mathrm{T}$ side to an $\mathrm{H}$ side and then to a $\mathrm{T}$ side of the other graphene sheet.

\section{ACKNOWLEDGMENT}

This work was supported by the Flemish Science Foundation (FWO-Vl) and the Methusalem Foundation of the Flemish Government.
[1] R. R. Nair, W. Ren, R. Jalil, I. Riaz, V. G. Kravets, L. Britnell, P. Blake, F. Schedin, A. S. Mayorov, S. Yuan, M. I. Katsnelson, H. M. Cheng, W. Strupinski, L. G. Bulusheva, A. V. Okotrub, I. V. Grigorieva, A. N. Grigorenko, K. S. Novoselov, and A. K. Geim, Small 6, 2877 (2010).

[2] X. Hong, S. H. Cheng, C. Herding, and J. Zhu, Phys. Rev. B 83, 085410 (2011).

[3] F. Withers, S. Russo, M. Dubois, and M. F. Craciun, Nanoscale Res. Lett. 6, 526 (2011).

[4] A. B. Bourlinos, K. Safarova, K. Siskova, and R. Zboril, Carbon 50, 1425 (2012).

[5] O. Leenaerts, H. Peelaers, A. D. Hernández-Nieves, B. Partoens, and F. M. Peeters, Phys. Rev. B 82, 195436 (2010).

[6] M. Klintenberg, S. Lebègue, M. I. Katsnelson, and O. Eriksson, Phys. Rev. B 81, 085433 (2010).

[7] J. T. Robinson, J. S. Burgess, C. E. Junkermeier, S. C. Badescu, T. L. Reinecke, F. K. Perkins, M. K. Zalalutdniov, J. W. Baldwin, J. C. Culbertson, P. E. Sheehan, and E. S. Snow, Nano Lett 10, 3001 (2010).

[8] R. Zboril, F. Karlicky, A. B. Bourlinos, T. A. Steriotis, A. K. Stubos, V. Georgakilas, K. Safarova, D. Jancik, C. Trapalis, and M. Otyepka, Small 6, 2885 (2010).

[9] D. K. Samarakoon, Z. Chen, C. Nicolas, and X. Q. Wang, Small 7, 965 (2011).

[10] A. Markevich, R. Jones, and P. R. Briddon, Phys. Rev. B 84, 115439 (2011).

[11] F. Withers, M. Dubois, and A. K. Savchenko, Phys. Rev. B 82, 073403 (2010).

[12] M. A. Ribas, A. K. Singh, P. B. Sorokin, and B. I. Yakobson, Nano Res. 4, 143 (2011).

[13] H. Y. Liu, Z. F. Hou, C. H. Hu, Y. Yang, and Z. Z. Zhu, J. Phys. Chem. C 116, 18193 (2012).

[14] N. Shen and J. O. Sofo, Phys. Rev. B 83, 245424 (2011).

[15] O. Leenaerts, B. Partoens, and F. M. Peeters, Phys. Rev. B 80, 245422 (2009).

[16] J. Sivek, O. Leenaerts, B. Partoens, and F. M. Peeters, J. Phys. Chem. C 116, 19240 (2012).

[17] H. F. Bettinger, K. N. Kudin, and G. E. Scuseria, J. Phys. Chem. A 108, 3016 (2004).

[18] M. S. Fuhrer, C. N. Lau, and A. H. MacDonald, MRS Bull. 35, 289 (2010).

[19] V. Singh, D. Joung, L. Zhai, S. Das, S. I. Khondaker, and S. Seal, Prog. Mater. Sci. 56, 1178 (2011).

[20] X. Li, G. Zhang, X. Bai, X. Sun, X. Wang, E. Wang, and H. Dai, Nat. Nanotech. 3, 538 (2008).
[21] K. S. Subrahmanyam, S. R. C. Vivekchand, A. Govindaraj, and C. N. R. Rao, J. Mater. Chem. 18, 1517 (2008).

[22] G. Eda and M. Chhowalla, Adv. Mater. 22, 2393 (2010).

[23] D. R. Dreyer, S. Park, C. W. Bielawski, and R. S. Ruoff, Chem. Soc. Rev. 39, 228 (2010).

[24] G. Eda, G. Fanchini, and M. Chhowalla, Nat. Nanotechnol. 3, 270 (2008).

[25] H. Kang, A. Kulkarni, S. Stankovich, R. S. Ruoff, and S. Baik, Carbon 47, 1520 (2009).

[26] A. Bagri, C. Mattevi, M. Acik, Y. J. Chabal, M. Chhowalla, and V. B. Shenoy, Nat. Chem. 2, 581 (2010).

[27] A. K. Kuriakose and J. L. Margrave, J. Phys. Chem. 69, 2772 (1965).

[28] Y. Kita, N. Watanabe, and Y. Fujii, J. Am. Chem. Soc. 101, 3832 (1979).

[29] K. A. Worsley, P. Ramesh, S. K. Mandal, S. Niyogi, M. E. Itkis, and R. C. Haddon, Chem. Phys. Lett. 445, 51 (2007).

[30] M. S. Dresselhaus and G. Dresselhaus, Adv. Phys. 51, 1 (2002).

[31] P. E. Blöchl, Phys. Rev. B 50, 17953 (1994); G. Kresse and D. Joubert, ibid. 59, 1758 (1999).

[32] G. Kresse and J. Hafner, Phys. Rev. B 47, 558 (1993); 49, 14251 (1994); G. Kresse and J. Furthmüller, Comp. Mater. Sci. 6, 15 (1996); Phys. Rev. B 54, 11169 (1996).

[33] S. S. Han, T. H. Yu, B. V. Merinov, A. C. Van Duin, R. Yazami, and W. A. Goddard, III, Chem. Mater. 22, 2142 (2010).

[34] J. P. Perdew, K. Burke, and M. Ernzerhof, Phys. Rev. Lett. 77, 3865 (1996).

[35] S. Grimme, J. Comp. Chem. 27, 1787 (2006).

[36] O. Leenaerts, B. Partoens, and F. M. Peeters, Appl. Phys. Lett. 93, 193107 (2008).

[37] J. O. Sofo, A. M. Suarez, G. Usaj, P. S. Cornaglia, A. D. Hernandez-Nieves, and C. A. Balseiro, Phys. Rev. B 83, 081411 (2011).

[38] H.-J. Kim and J.-H. Cho, Phys. Rev. B 87, 174435 (2013).

[39] Richard F. W. Bader, Atoms in Molecules - A Quantum Theory (Oxford University Press, Oxford, 1990).

[40] G. Henkelman, A. Arnaldsson, and H. Jónsson, Comput. Mater. Sci. 36, 354 (2006).

[41] A. C. T. van Duin, S. Dasgupta, F. Lorant, and W. A. Goddard, J. Phys. Chem. A 105, 9396 (2001).

[42] S. K. Singh, S. Goverapet Srinivasan, M. Neek-Amal, S. Costamagna, Adri C. T. van Duin, and F. M. Peeters, Phys. Rev. B 87, 104114 (2013).

[43] S. K. Singh, S. Costamagna, M. Neek-Amal, and F. M. Peeters, J. Phys. Chem. C 118, 4460 (2013). 